\title{
ESTUDO DA MORFOLOGIA, TAMANHO, DISTRIBUIÇÃO E PRECIPITAÇÃO SOBRE ÓXIDOS DE INCLUSÕES DE SULFETOS DE MANGANÊS EM AÇOS RESSULFURADOS
}

\author{
Alexsandro Bobsin ' \\ Wagner Viana Bielefeldt ' \\ Antônio Cezar Faria Vilela'
}

\section{Resumo}

O enxofre tem influência direta nas propriedades mecânicas nas diversas classes de aços, principalmente se formar FeS que reduz a resistência no trabalho a quente, devido ao seu baixo ponto fusão. A fim de que o teor de enxofre seja controlado na produção do aço, tem sido aplicada a adição de elementos de liga como o manganês. $O$ manganês reduz consideravelmente a solubilidade do enxofre pela formação de sulfetos de manganês com a redução da temperatura. Estas inclusões controlam o crescimento do grão e facilitam a usinagem, aumentando a vida útil das ferramentas de corte. No entanto, prejudicam a qualidade dos aços e provocam problemas como redução na dutilidade e qualidade superficial de chapas laminadas a quente. Portanto, este trabalho fará uma abordagem sobre as influencias de processos, objetivando estudar sua morfologia, distribuição e tamanho médio, assim como, a precipitação dos sulfetos de manganês sobre óxidos; usando análise de imagens produzidas a partir de amostras retiradas de aço ressulfurado.

Palavras-chave: Inclusões não metálicas; Sulfetos de manganês; Óxidos nucleantes.

\section{STUDY OF MORPHOLOGY, SIZE, DISTRIBUTION AND PRECIPITATION ON OXIDES OF INCLUSIONS OF MANGANESE IN RESULPHURISED STEELS}

\begin{abstract}
Sulfur has a direct influence on the mechanical properties of the various grades of steel, especially if it forms FeS, which reduces the resistance to hot rolling due to its low melting point. In order that the sulfur content is controlled in the production of steel, the addition of alloying elements such as manganese has been applied. Manganese considerably reduces the solubility of sulfur by the formation of manganese sulphides with the reduction of temperature. These inclusions control grain growth and facilitate machining, increasing the life of cutting tools. However, they impair the quality of the steels and cause problems such as reduction in the ductility and surface quality of hot-rolled sheets. Therefore, this work will focus on the influences of processes, aiming to study their morphology, distribution and average size, as well as the precipitation of manganese sulphides on oxides; using image analysis produced from samples taken from resulphurised steel. Keywords: Non-metallic inclusions; Manganese sulfides; Oxide seed.
\end{abstract}

\section{INTRODUÇÃO}

A presença de sulfetos de manganês geralmente é prejudicial para as propriedades mecânicas da maioria dos aços, pois, sua composição, forma, tamanho e distribuição afetam as propriedades mecânicas, de corrosão e trabalho a quente, de acordo com Ken-ichi Yamamoto et al. [I]. No entanto, sua presença em aços ressulfurados ou de usinagem fácil tem cada vez mais encontrando aplicações na indústria. Entender o comportamento sulfetos de manganês é de fundamental importância para garantir que sejam atendidas as crescentes exigências de qualidade, principalmente do setor automobilístico. Desta forma, Mapelli et al. [2] aborda em seu trabalho o controle da formação destas inclusões, baseadas em modelos das reações de equilíbrio, usando dados e diferentes taxas de aquecimento durante vários estágios do processo secundário de refino, analisando a morfologia e composição dos sulfetos de manganês. Portanto, a revisão da literatura deste trabalho tem o intuito de esclarecer os mecanismos de formação destas inclusões não metálicas,

'Laboratório de Siderurgia - LaSid, Programa de Pós-graduação em Engenharia de Minas, Metalúrgica e de Materiais - PPGE3M, Departamento de Metalurgia, Universidade Federal do Rio Grande do Sul - UFRGS, Porto Alegre, RS, Brasil. E-mail: abobsin@gmail.com 
não só em aços ressulfurados C-Mn acalmado ao alumínio, mas também abordar outros tipos de aços, de forma a se ter uma compreensão mais completa destas. Serão estudadas as influencias de processo na morfologia, distribuição e diâmetro médio destas inclusões através de microscopia eletrônica de varredura (MEV). Também será estudado o comportamento da precipitação de sulfetos de manganês sobre óxidos, utilizando espectroscopia de energia dispersiva (EDS), afim de que, se possam compreender quais os principais óxidos nucleantes em aço ressulfurado.

\section{DESENVOLVIMENTO}

\section{I Formação dos Sulfetos de Manganês}

No trabalho de Oikawa et al. [3] é encontrada a morfologia e classificação dos tipos de sulfetos de manganês segundo Sims [4]. Os mais comuns de se observarem são os de forma globular (tipo I - Figura la), eutético (tipo II - Figura Ib), dendrítico (Figura Ic) e angular (tipo III - Figura Id). As demais morfologias são variações destas, dependendo do próprio teor de enxofre e da presença de elementos de liga, segundo Oikawa et al. [3].

Para entender a formação termodinâmica de sulfetos de manganês, Meng-long Li et al. [5] analisou um diagrama pseudobinário (Fe-MnS) como a Figura 2.

A reação eutética $L I \rightarrow \delta \mathrm{Fe}+\mathrm{MnS}$ (ponto e da Figura 2) fará precipitar sulfetos de manganês eutéticos (tipo II) no contorno de grão. Para que ocorram sulfetos do globular (tipo I) é necessária uma reação monotética tido como, $\mathrm{LI} \rightarrow \mathrm{Fe}(\mathrm{s})+\mathrm{L} 2$ enriquecido em MnS (linha e- $x$ da Figura 2). Os do tipo III angular só ocorrem com maiores teores de $\mathrm{MnS}$ superiores a 0,015\% em massa (linha e-y da Figura 2). De acordo com o mesmo autor, quando a fração sólida de Fe alcança $98,5 \%$, cerca de $87 \%$ do $\mathrm{MnS}$ precipita, porque no líquido residual o produto de solubilidade de $[\mathrm{Mn}][\mathrm{S}]$ é maior que no equilíbrio. Isto também pode ser visto no trabalho de Peng-ju Chen et al. [6] que fez uma análise termodinâmica da precipitação do sulfeto de manganês em aço rolamento. $O$ resultado pode ser visto na Figura 3.

No que se refere a influencia do contorno de grão, alguns trabalhos analisaram o comportamento de sulfetos de manganês desde a solidificação na ferrita delta, como
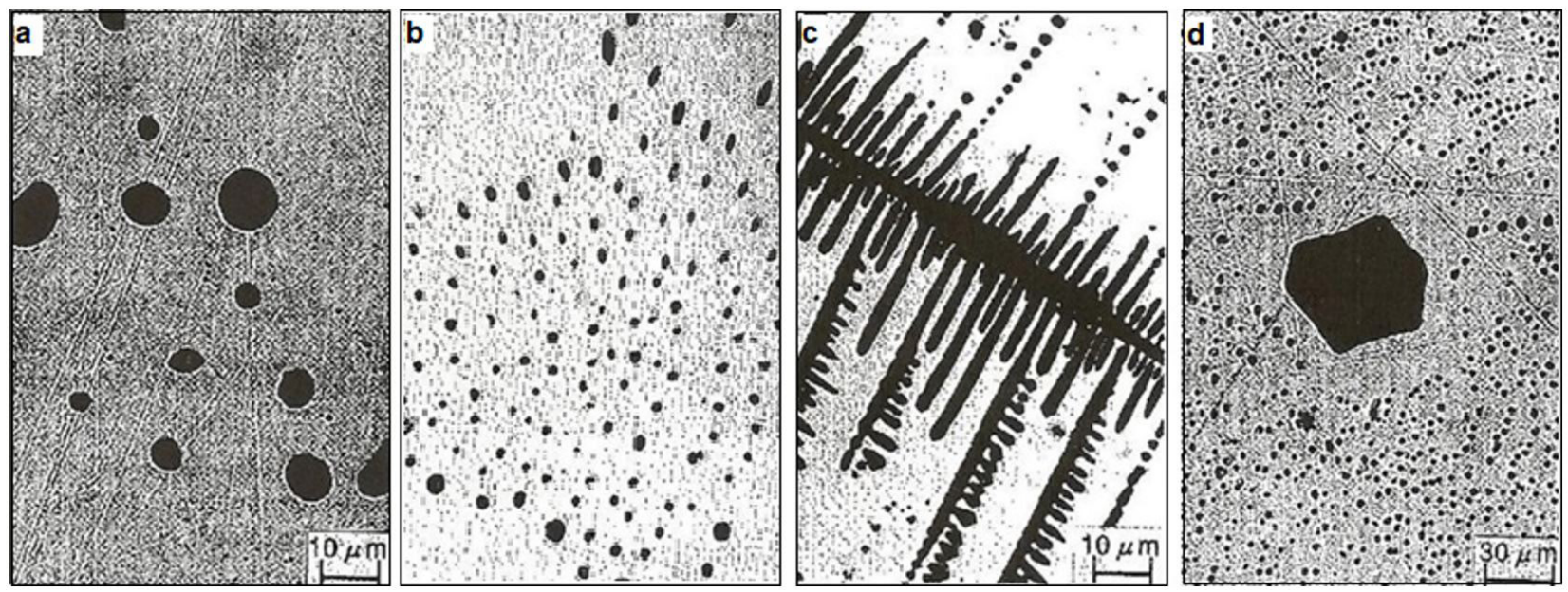

Figura I. Morfologia de sulfetos: (a) globular (tipo I); (b) eutético em partículas (tipo II); (c) dendrítico; (d) angular (tipo III) (Oikawa et al. [3]).

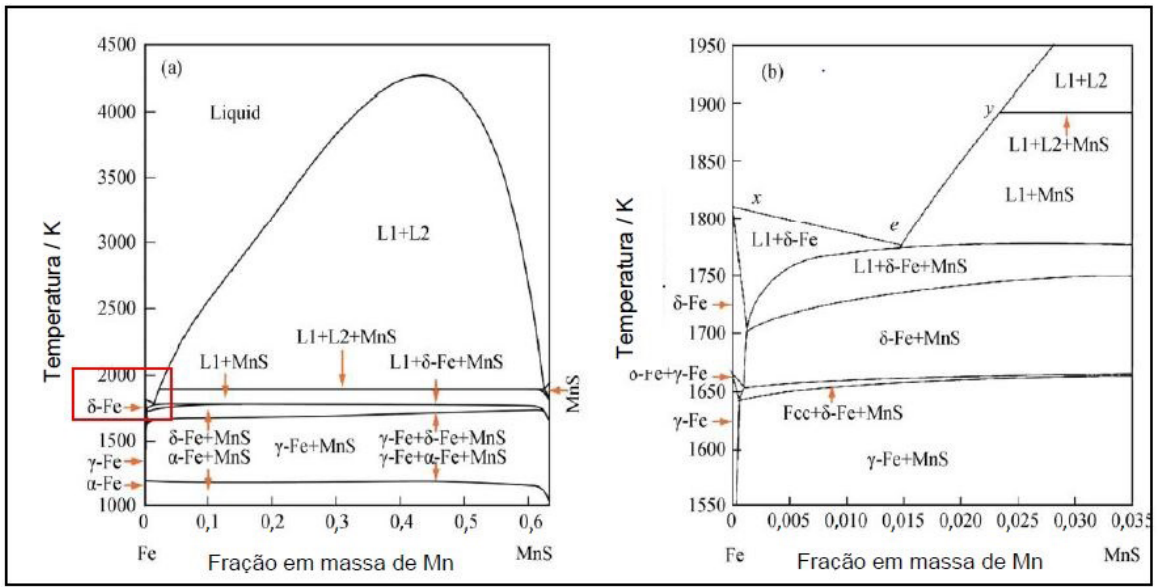

Figura 2. (a) Fe-MnS diagrama de fase pseudo-binário (b) magnificação na região entre próximo a I00\% Fe (Meng-long Li et al. [5]). 


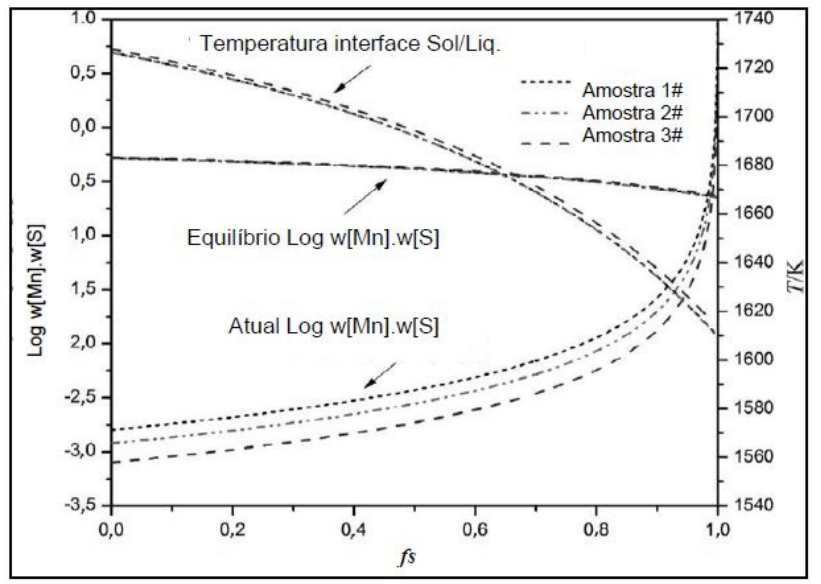

Figura 3. Produto de solubilidade do $\mathrm{MnS}$ variando com a fração sólida (Peng-ju Chen et al. [6]).

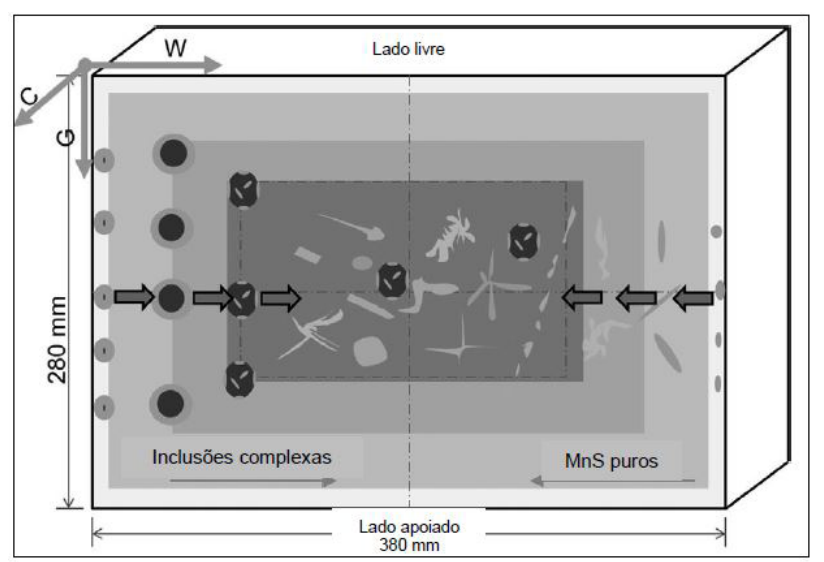

Figura 4. Morfologia dos MnS no lingote (Xuewei Zhang et al. [7]).

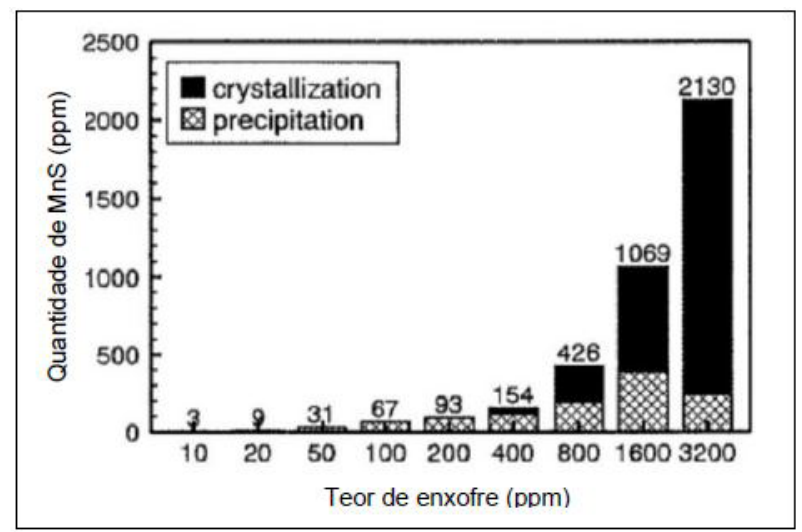

Figura 5. Variação da precipitação e cristalização em função da variação do teor de S (Wakoh et al. [13]).

o de Xuewei Zhang et al. [7], passando por precipitados nanométricos de $\mathrm{MnS}$ no grão austenítico no estudo de $\mathrm{Yu}$ Hao et al. [8]. A influência do espaço dendrítico foi abordado por Imagumbai [9] que descreveu modelos de crescimento do braço dendrítico primário e secundário, porque estes influenciam na morfologia e tamanho dos sulfetos. Diederichs e Bleck [10] propôs outro modelo focado no enriquecimento do líquido residual entre as dendritas.

No trabalho de Xuewei Zhang et al. [7] que usou lingotes de aço trilho como amostra, foi constatado que a morfologia das inclusões de sulfetos de manganês, que parte de esférica e elíptica mais próxima à superfície, mudando para alongada, poliédrica e irregular, em direção ao centro gradualmente, de acordo com a Figura 4 .

A distribuição e tamanho das inclusões dos sulfetos de manganês são influenciados por uma série de variáveis, desde o metal líquido, passando pelo processo de refino secundário até o resfriamento[I I ] e solidificação do lingote. Desta forma, X.F. Zhang et al. [12] constatou que o uso de pulsos elétricos durante o banho metálico foi muito eficaz na redução do tamanho e distribuição dos $\mathrm{MnS}$; baseado na diferença de condutividade entre inclusão e fundido metálico.

No refino secundário, existem muitos trabalhos dedicados ao estudo de óxidos como sítios nucleantes para sulfetos de manganês, pois segundo Oikawa et al. [3], sulfetos globulares (tipo I) se formam no banho metálico.

$O$ teor de enxofre e elementos de liga influenciam não só na precipitação, como também na morfologia do MnS. De acordo com Wakoh et al. [13], para baixos teores de enxofre, a precipitação (formação da inclusão MnS heterogênea) é maior que a cristalização (formação da inclusão pura de $\mathrm{MnS}$ ), porém a partir de 400 ppm de enxofre a cristalização começa a ser maior que a precipitação, como mostra Figura 5.

Avaliando-se a solidificação, no trabalho de lto et al. [14] foram produzidas amostras em laboratório com diferentes taxas de resfriamento. Ficou constatado que, quanto maior a taxa de resfriamento, menor $\circ$ tamanho da dendrita e consequentemente, mais enriquecido o líquido residual entre elas, resultando um tamanho menor dos $\mathrm{MnS}$ e um aumento na densidade (refino) dos mesmos.

$\mathrm{Na}$ avaliação de amostras industriais no lingotamento de aço de trilho UV75 feito por Sen Luo et al. [15] na Figura 6 , os sulfetos tiveram tamanhos refinados e dispersos na região coquilhada (SA) onde a taxa de resfriamento é alta. À medida que se avançou para zona colunar (CA), mista (MA) e equiaxial (EA); o tamanho das inclusões de sulfetos de manganês aumentou, reduzindo a densidade e modificando sua morfologia de globular para alongadas. Foi considerado também o tamanho do espaço dendrítico, concluindo que à medida que este reduz, os sulfetos também reduzem o tamanho e ficando mais distribuídos.

Inclusões alongadas de sulfetos de manganês reduzem as propriedades mecânicas de muitos aços. Após a laminação a quente, podem se alinhar; podendo propagar trincas e formar vazios, que coalescendo, reduzem a ductilidade local, como indicou Ken-ichi Yamamoto et al. [I] na Figura 7. Seu estudo foi com aços do tipo TS, submetidos a diferentes teores de enxofre e temperaturas de reaquecimento de 
laminação SRT. Após estes foram submetidos a ensaio de tração para analisar a redução de área. A que apresentou pior desempenho foi TS 94, pelo maior teor de enxofre e baixa temperatura de laminação, porque as inclusões alongadas não dissolveram.

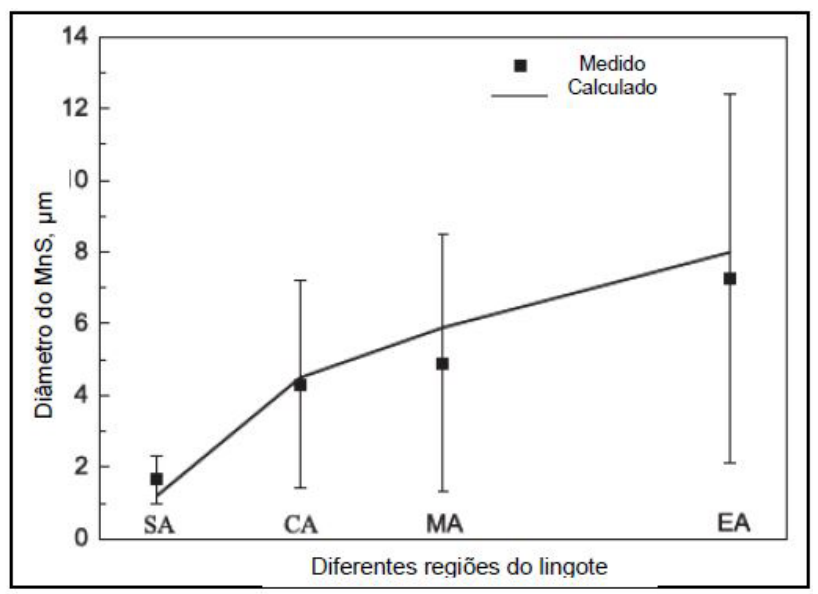

Figura 6. Comparação entre diâmetro equivalente medido e diâmetro médio calculado da inclusão de $\mathrm{MnS}$ em diferentes regiões do corte transversal do lingote (Sen Luo et al. [15]).

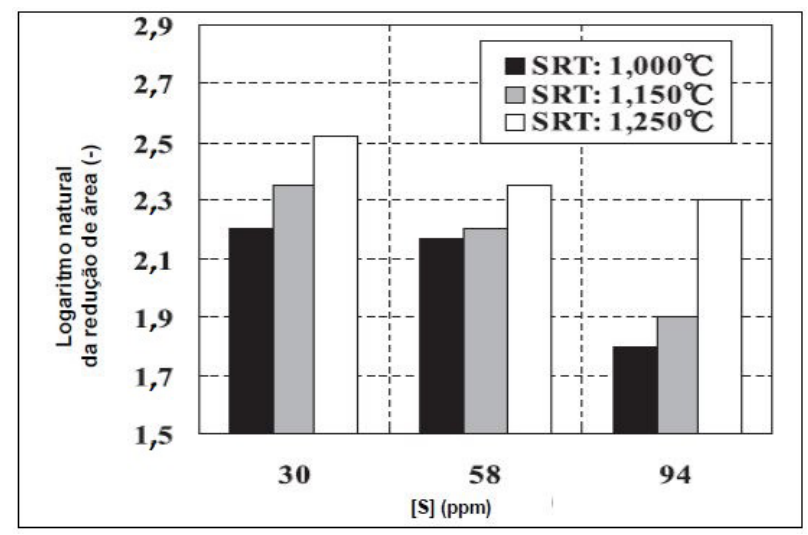

Figura 7. Variação da redução de área pelo teor de enxofre nas temperaturas de reaquecimento (Ken-ichi Yamamoto et al. [I]).

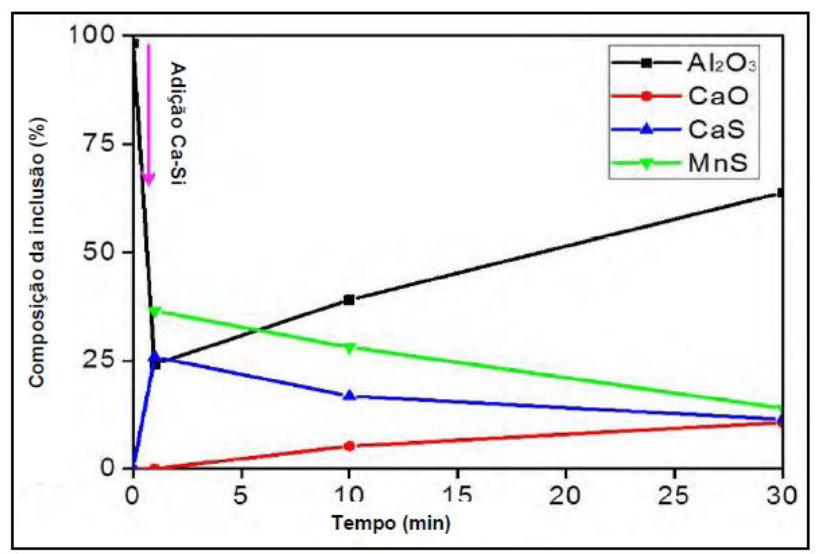

Figura 8. Evolução da composição das inclusões (Ying Ren [18]).
Para melhorar a morfologia dos sulfetos alongados, o estudo proposto por Xiaojing Shao et al. [16] utilizou tratamento térmico e mostrou que quanto maior a temperatura ( $1463 \mathrm{~K})$ e tempo de imersão (4 horas), aliado a uma baixa taxa de aquecimento (menor que $2 \mathrm{~K} / \mathrm{min}$ ) se mostrou eficaz no tratamento de inclusões alongadas, pois, houve uma partição das mesmas e eventual esferiodização.

\subsection{Influência dos Óxidos na Distribuição dos Sulfetos de Manganês}

Esta abordagem será dedicada ao estudo de inclusões de óxidos como sítios nucleantes de sulfeto de manganês. Sendo assim, é necessário entender a formação destes óxidos no refino secundário, principalmente a alumina $\left(\mathrm{Al}_{2} \mathrm{O}_{3}\right)$ que é um dos principais agentes de nucleação para sulfetos de manganês. No entanto, este óxido pode ser modificado através da adição de cálcio para inclusões líquidas complexas do sistema $\mathrm{CaO}-\mathrm{Al}_{2} \mathrm{O}_{3}$ (cálcio aluminatos-CA) que servem de núcleo para precipitação, conforme Bielefeldt e Vilela [17] ou de $\mathrm{CaO}-\mathrm{MgO}-\mathrm{Al}_{2} \mathrm{O}_{3}(\mathrm{CMA})$ por Peng-ju Chen et al. [6]. Além disso, o cálcio funciona como um redutor no teor de enxofre no banho metálico pela formação de CaS, de acordo com Imagumbai e Takeda [18]. Portanto, para se entender como o MnS precipita sobre óxidos; é necessário entender a formação destas inclusões no banho líquido em diferentes tipos de aço.

De acordo com Ying Ren et al. [19], é de conhecimento que as inclusões de $\mathrm{CaS}$ são geradas como uma fase de transição, a partir da adição de cálcio, que dissolvido junto com o enxofre, forma $\mathrm{CaS}$, modificando inclusões de $\mathrm{Al}_{2} \mathrm{O}_{3}$ ou $\mathrm{MgO}-\mathrm{Al}_{2} \mathrm{O}_{3}$ para cálcio-aluminatos, como mostrado nas reações I a 3:

$$
\begin{aligned}
& {[\mathrm{Ca}]+[\mathrm{S}] \rightarrow \mathrm{CaS}} \\
& 3 \mathrm{CaS}+\mathrm{Al}_{2} \mathrm{O}_{3} \rightarrow 3(\mathrm{CaO})+2[\mathrm{Al}]+3[\mathrm{~S}] \\
& \mathrm{xCaS}+\mathrm{xMgO} \cdot \mathrm{yAl}_{2} \mathrm{O}_{3} \rightarrow \mathrm{xCaO} \cdot \mathrm{yAl}_{2} \mathrm{O}_{3}+x[\mathrm{Mg}]+\mathrm{x}[\mathrm{S}]
\end{aligned}
$$

Inclusões de $\mathrm{Al}_{2} \mathrm{O}_{3}$ angulares são geradas no processo de desoxidação. Após a adição de $\mathrm{Ca}$-Si, o cálcio reage com o enxofre para produzir CaS. Este por sua vez, precipita na superfície angular do $\mathrm{Al}_{2} \mathrm{O}_{3}$ formando uma inclusão complexa esférica devido a energia interfacial livre. Alcançando-se o equilíbrio, a camada de $\mathrm{CaS}$ reage com o núcleo de $\mathrm{Al}_{2} \mathrm{O}_{3}$ ou oxigênio para formar o cálcio-aluminato. A adição de $\mathrm{Ca}-\mathrm{Si}$ reduz a quantidade de $\mathrm{Al}_{2} \mathrm{O}_{3}, \mathrm{MnS}$ e $\mathrm{CaS}$, como mostra Figura 8.

Além disso, Bielefeldt e Vilela [I7] analisou a modificação da alumina por adição de diferentes teores de cálcio em aço SAE 8620 a I $813 \mathrm{~K}\left(1540^{\circ} \mathrm{C}\right)$, concluindo que há um aumento na janela líquida, isto é, as inclusões são modificadas para o estado líquido. No trabalho de Y. Hu et al. [20] fica demonstrado que inclusões do sistema $\mathrm{Al}_{2} \mathrm{O}_{3}-\mathrm{SiO}_{2}-\mathrm{CaO}-$ $\mathrm{MgO}-\mathrm{CaS}$, reduziram seu ponto de fusão; muitas inclusões 

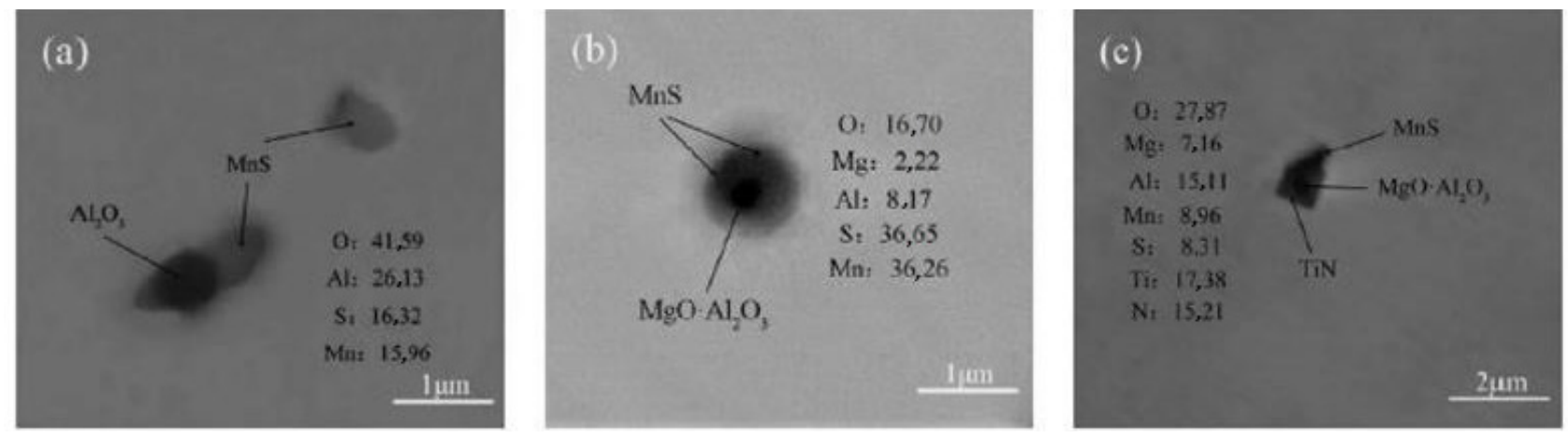

Figura 9. (a) $\mathrm{Al}_{2} \mathrm{O}_{3}$ encapsulado com MnS; (b) MA encapsulado com MnS e (c) MA encapsulado com MnS e TiN (Peng-ju Chen et al. [6]).

migraram da temperatura de fusão de $1500^{\circ} \mathrm{C}-1700^{\circ} \mathrm{C}$ para $1400^{\circ} \mathrm{C}-1500^{\circ} \mathrm{C}$, por adição de $\mathrm{Ca}$.

Peng-ju Chen et al. [6] estudou a precipitação de sulfetos de manganês sobre óxidos MA e CMA. O trabalho mostrou como o teor de enxofre interfere na precipitação dos sulfetos de manganês sobre óxidos complexos, em sua morfologia e tamanho. Foi observado que a maior quantidade de inclusões não metálicas são os óxidos-sulfetos. Os tipos de óxidos e a concentração de enxofre nas amostras, indicam a proporção de $\mathrm{MnS}$ precipitados sobre estes óxidos. A morfologia e a composição química das inclusões de sulfetos de manganês, contendo inclusões, nas amostras metalográficas podem ser vistas na Figura 9.

Baseado em outros estudos, ficou demonstrado que a modificação da $\mathrm{Al}_{2} \mathrm{O}_{3}$ pela presença de $\mathrm{Mg}$, formando inclusões que seguem o seguinte: $M A \rightarrow C M A \rightarrow C A$, sendo que o CA precisa de um tempo considerável. Assim, Peng-ju Chen et al. [6] demonstra que taxa de precipitação e espessura média dos sulfetos de manganês, sobre os MA e CMA, diminui na mesma relação de redução do teor de enxofre. Além disso, a taxa de precipitação é maior sobre as inclusões de MA do que CMA. As espessuras médias dos MnS sobre CMA são de 2.5 a 4.5 maior em relação as de MA, como mostra a Figura 10.

\section{MATERIAIS E MÉTODOS}

O aço estudado neste trabalho é um aço ressulfurado do tipo SAE I I 4 I, cuja composição química média das 4 corridas é mostrada na Tabela I. Como se pode observar na tabela, é um aço com baixos teores de elementos de liga com exceção do manganês que possui um teor maior para formação dos sulfetos de manganês.

O aço ressulfurado foi produzido em forno elétrico a arco (FEA), seguido de forno-panela. No próximo passo, a panela segue para a estação de desgaseificação a vácuo (VD). Finalizando, o metal liquido é levado para estação de lingotamento para formação dos tarugos, seguindo para laminação à quente de barras.

A amostragem ocorreu no final do lingotamento contínuo, após a solidificação dos tarugos. Cada amostra

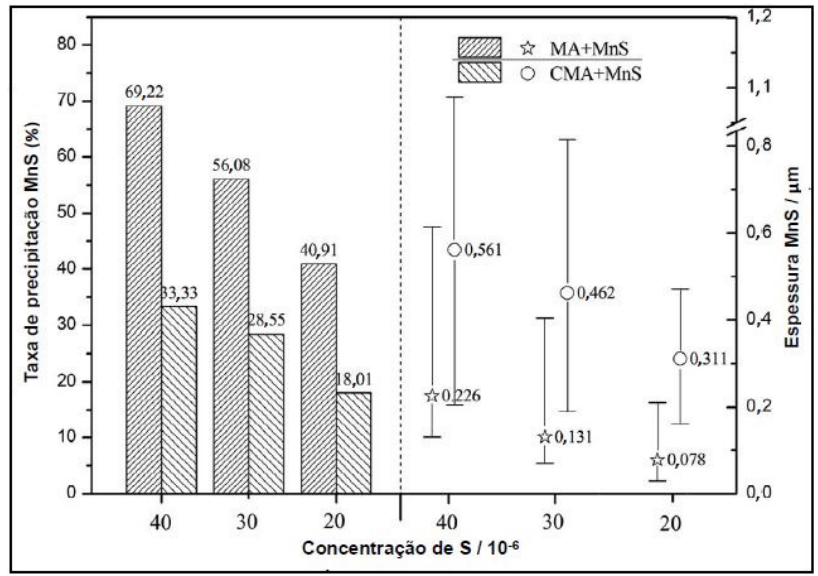

Figura 10. Efeito do enxofre na proporção de precipitação e espessura do $\mathrm{MnS}$ nas inclusões MA e CMA (Peng-ju Chen et al. [6]).

Tabela I. Composição química média do aço estudado neste trabalho SAE II4I

\begin{tabular}{ccccccccc}
\hline Elementos & $\mathbf{C}$ & $\mathbf{S i}$ & $\mathbf{M n}$ & $\mathbf{S}$ & $\mathbf{C r}$ & $\mathbf{N i}$ & $\mathbf{A l}$ & $\mathbf{C a}$ \\
\hline 0,39 & 0,16 & $\mathrm{I}, 5 \mathrm{I}$ & 0,094 & 0,16 & 0,08 & 0,021 & 0,0006 \\
\hline
\end{tabular}

de aço foi coletada da seguinte maneira: uma em meio raio $(\mathrm{I} / 2)$ e uma do núcleo $(\mathrm{N})$, sendo o número ao lado de $(\mathrm{A})$ indica o número da corrida, por exemplo: Corrida I amostra: $\mathrm{Al}(\mathrm{I} / 2)$ e Al (N). As amostras coletadas do tarugo foram cortadas com disco de corte refrigerado na seção representada na Figura II, seguindo de processo metalográfico de lixamento, polimento.

$A$ análise via MEV/EDS foi utilizado um aparelho marca JEOL modelo JSM 5800, com EDS acoplado, localizado no Centro de Microscopia Eletrônica (CME-UFRGS). Os parâmetros de análise no EDS foram: ângulo entre a superfície e o feixe de elétrons de $90^{\circ}$; voltagem para aceleração de elétrons entre 15 e $20 \mathrm{kV}$; tempo de contagem de $100 \mathrm{~s}$; dead time abaixo de $40 \%$. O MEV foi utilizado para análise da morfologia, dimensão, análise química e mapeamento químico das inclusões. A superfície de $200 \mathrm{~mm}^{2}$ foi analisada de cada amostra, para encontrar os tipos de sulfetos e sua distribuição nessa seção. As inclusões mais características foram fotografadas, sendo algumas analisadas 
e mapeadas quimicamente. Para analisar o tamanho médio das inclusões e distribuição destas, utilizou-se o programa Image J. Os parâmetros de preparação do programa para análise das figuras foram determinados para análise da dispersão e tamanho dos sulfetos.

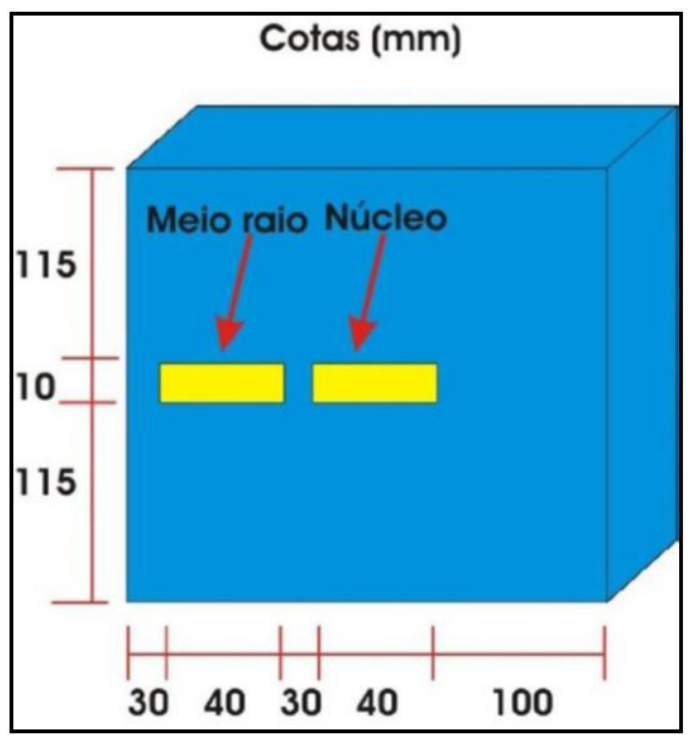

Figura II. Local do tarugo e a dimensão das amostras analisadas no MEV/EDS.
O processamento envolveu duas imagens (aumento de $100 \mathrm{X}$ ), sendo uma de meio raio e outra do núcleo, nas quatro corridas. No total foram visualizadas quarenta micrografias.

\section{RESULTADOS E DISCUSSÃO}

\section{I Morfologia das Inclusões de Sulfetos de Manganês}

O estudo das imagens produzidas no MEV são mostradas as morfologias mais encontradas nas amostras, conforme a Figura 12.

Foi constatado que na região de meio raio, o tipo de formação mais encontrado foi do tipo III (angular) ou do tipo irregular, havendo também a presença, em menor número, do tipo Il (eutético em partículas) como mostra a Figura 12. A formação dos sulfetos tipo III, como já foi discutido no subitem 2.I, está possivelmente ligado a uma reação no estado sólido peritética Meng-long Li et al. [5]. A formação dos sulfetos irregulares ocorre pela perda da forma do tipo III, na transição da austenita para ferrita. Os sulfetos circulares, possivelmente sejam do tipo II em partículas, pois os sulfetos globulares e esféricos são maiores.

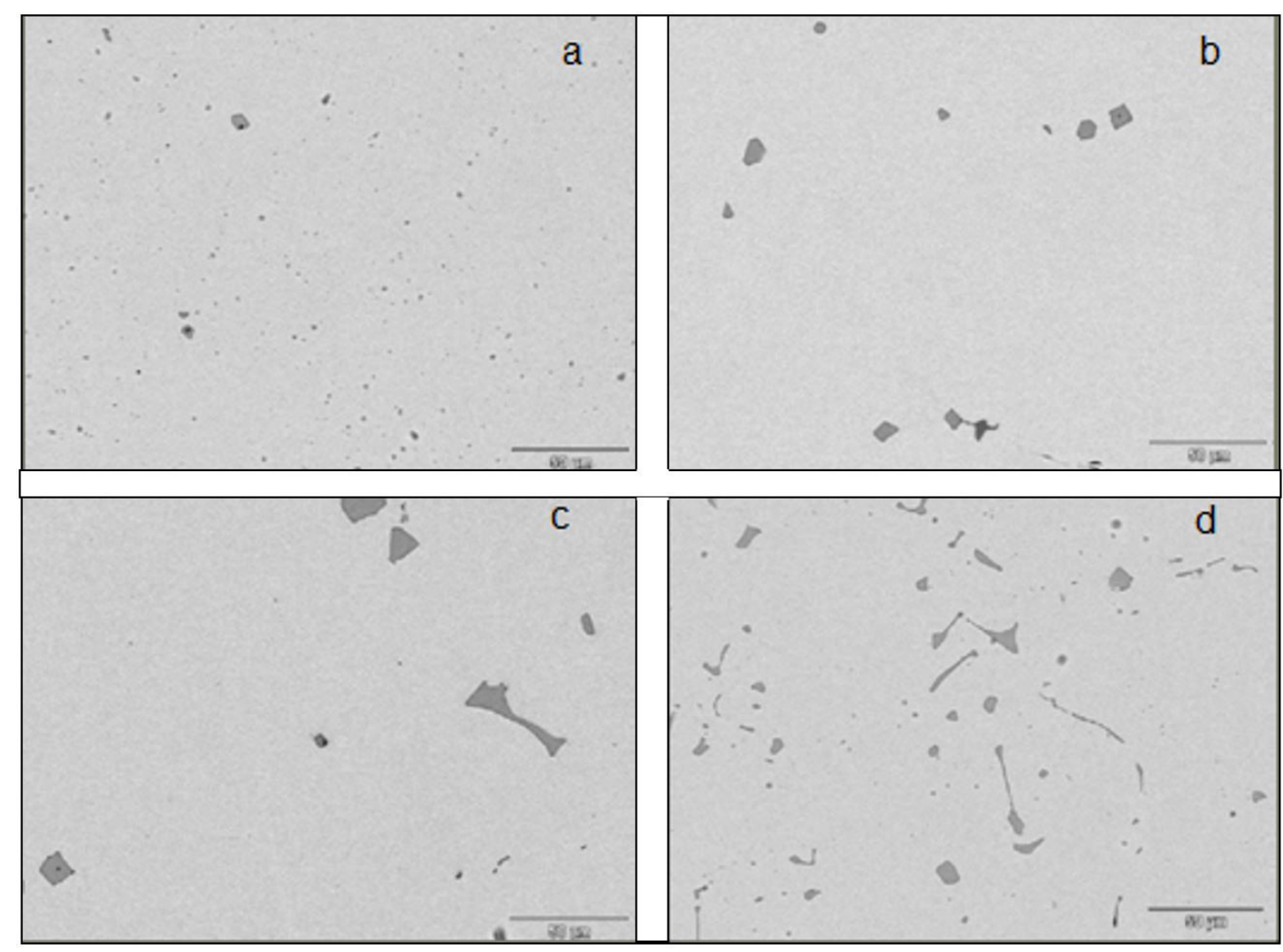

Figura 12. (a) MnS eutético em partículas, $A 4$ (I/2) de meio raio (b) MnS angular, A3 (I/2) de meio raio (c) MnS irregular, Al (I/2) meio raio (d) MnS eutético e em placas, AI (N) do núcleo. 


\subsection{Distribuição das Inclusões de Sulfetos de Manganês.}

Com todos os dados levantados foi feita uma média das quatro amostras, das diferentes corridas, obtendo-se os valores médios de acordo com a Tabela 2 .

Quando se verificam as micrografias, as análises, os gráficos e dados gerados, foram feitas algumas considerações:

- Em geral o número de inclusões aumentou em direção ao núcleo do tarugo. Isto está de acordo com o trabalho de lto et al. [14] e Sen Luo et al. [15] demonstrado na Figura 6 do subitem (2.I). Isto pode ser explicado pelo fato da região do núcleo apresentar um teor de enxofre maior, favorecendo assim a precipitação de um número maior de inclusões. Além da evidência visual das micrografias que há maior concentração de sulfetos do tipo II (eutético) ou eutético irregular no núcleo. Verificou-se que a circularidade, que é medida de acordo com a parametrização do software de imagens; diminui em direção ao núcleo, sendo uma comprovação de que as inclusões ficam mais alongadas, também o que foi encontrado no artigo de Xuewei Zhang et al. [7];

- O diâmetro e o tamanho médio das inclusões diminuem em direção ao núcleo, com exceção da amostra Al. Isto está em desacordo com o subitem 2.I que diz que se o espaçamento interdendrítico aumentar, haveria um incremento no diâmetro da inclusão, segundo Meng-long Li et al. [5] e Ito et al. [14]. Porém isso pode ser explicado também pelo fato do aumento do número de inclusões na região do centro do tarugo. No trabalho de Imagumbai [9], isto pode ser constatado

Tabela 2. Análise de distribuição das inclusões de sulfeto de manganês

\begin{tabular}{lcc}
\hline \multicolumn{1}{c}{ Item } & Meio raio & Núcleo \\
\hline Média de inclusões $/ \mathrm{mm}^{2}$ & 73 & 130 \\
Fração de área média $(\%)$ & 0,47 & 0,55 \\
Tamanho médio $\left(\mu \mathrm{m}^{2}\right)$ & 65,38 & 52,87 \\
Diâmetro médio $(\mu \mathrm{m})$ & 13,47 & 12,88 \\
\hline
\end{tabular}

mais claramente, pois o número de inclusões aumenta e o diâmetro médio é reduzido;

- Na região de meio raio existe uma predominância maior de sulfetos do tipo III (angular) ou irregular como encontrada na Figura 4 de Xuewei Zhang et al. [7]. Estas inclusões predominam na zona colunar e mista, principalmente no lado de lingotamento do tarugo. Contata-se isto pelo fato do diâmetro médio e a circularidade serem maiores nestas regiões, comprovando que estas inclusões possuem uma forma mais geométrica. Também se verifica que 0 número de sulfetos maiores que $40 \mu \mathrm{m}$, que é o tamanho médio dos sulfetos tipo III (angulares) e irregulares, se manteve aproximadamente igual em número.

\section{3 Óxidos como Sítios Nucleantes de Sulfetos de Manganês}

Como já foi comentado no subitem 2.2, óxidos podem funcionar como sítios de nucleação para a precipitação de sulfetos de manganês. Estes auxiliariam uma nucleação heterogênea na fase líquida, favorecendo a dispersão dos sulfetos na fase sólida. Através disso, deve-se considerar o tipo de óxido, à distância entre estes e a quantidade, conforme Wakoh et al. [13]. Neste estudo tem-se o interesse em verificar esta teoria, indicando o provável tipo de óxido que esta funcionando como nucleante, através de mapeamento e análise química via EDS. Deve-se considerar que a análise química por EDS da uma estimativa dos elementos presentes na região dos óxidos investigados. Portanto, foram procuradas inclusões de sulfetos que continham em seu interior óxidos. Um exemplo disto está mostrado na Figura I3. Nesta tem-se um sulfeto do tipo irregular precipitado sobre o óxido de alumínio, pelo que indica o espectro e a análise química do óxido, demostrando na tabela 3.

A inspeção demonstrou que os sulfetos do tipo III (angular) e do tipo irregular são as mais frequentes inclusões que possuem um óxido. Isto foi mostrado no artigo de Xuewei Zhang et al. [7] do item (2.I). Entretanto; foram
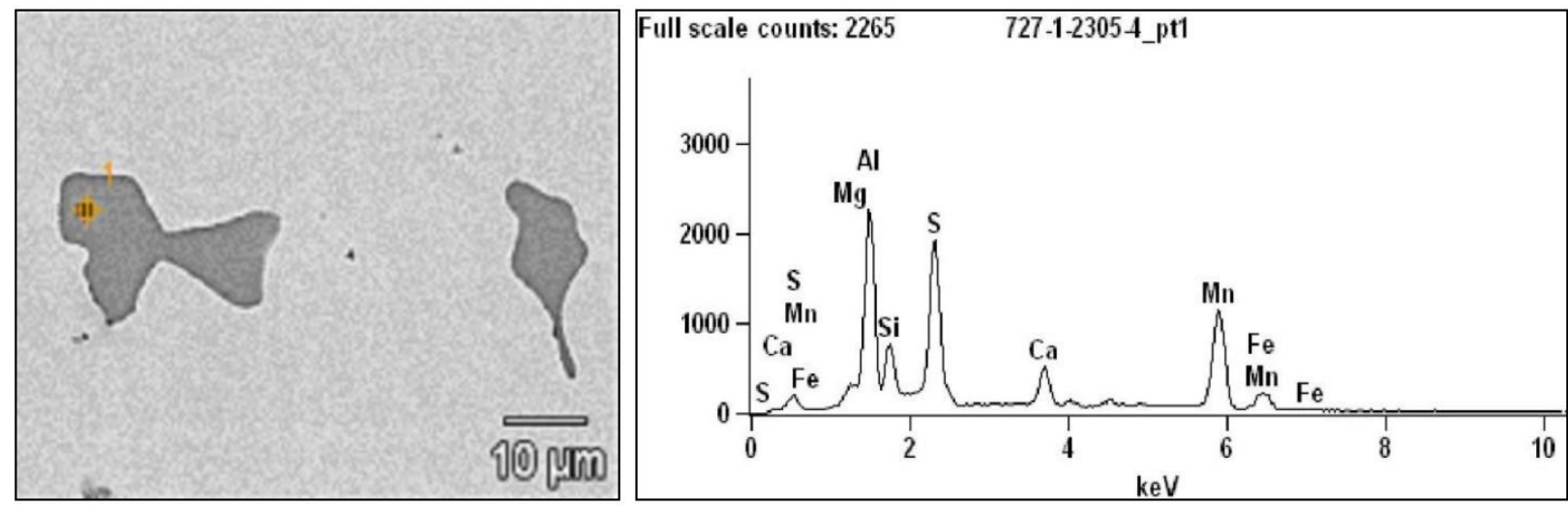

Figura 13. Sulfeto irregular precipitado sobre óxido de $\mathrm{Al}_{2} \mathrm{O}_{3}$. 
encontradas algumas inclusões que possuem uma morfologia próxima do tipo I (globular) como pode ser conferido na Figura 14, concordando como foi mostrado na Figura 9 de Peng-ju Chen et al. [6]. Pelo espectro e análise de EDS, possivelmente o óxido seja de cálcio alumínio e silício, como mostra a tabela 4 .

Com intuito de demonstrar que o sulfeto está nucleado sobre um óxido, foi realizado a análise química sobre a área do óxido (ponto I) e do sulfeto (ponto 2) ilustrado na Figura 15. O espectro mostra pelos teores que se trata de um sulfeto. A análise química na Tabela 5 reforça a ideia de se tratar de um sulfeto associado a um óxido de cálcio e alumínio.

Para ver a distribuição de cada elemento químico foi traçado um mapa que se encontra na Figura 16. O mapeamento químico analisa o $\mathrm{Mg}, \mathrm{Al}, \mathrm{Mn}, \mathrm{Si}, \mathrm{S}, \mathrm{Ca}$, e Fe. Por isso, pela presença de $\mathrm{Mg}$, Si e Al; demonstra que o provável óxido se trata de um cálcio aluminato com silício, rodeado por um anel de $\mathrm{MnS}$, confirmando o que foi dito por Ying Ren et al. [19] e Peng-ju Chen et al. [6].
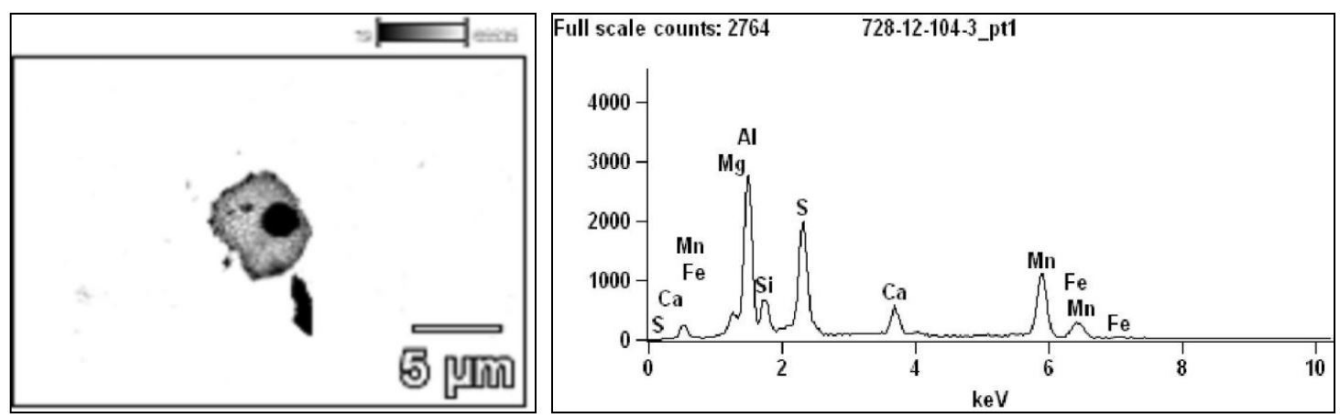

Figura 14. Imagem mostra a inclusão de sulfeto com óxido. O espectro resultante da análise química pontual por EDS no ponto I assinalado na imagem.
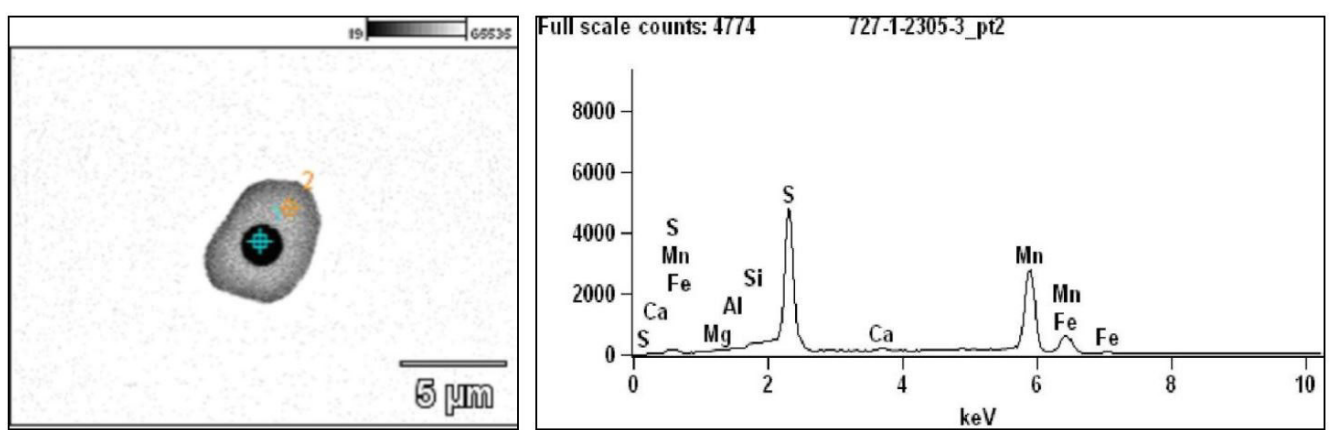

Figura I5. Imagem mostra a inclusão de sulfeto com óxido à direita. $\mathrm{O}$ espectro resultante da análise química pontual por EDS no ponto 2 assinalado na imagem.
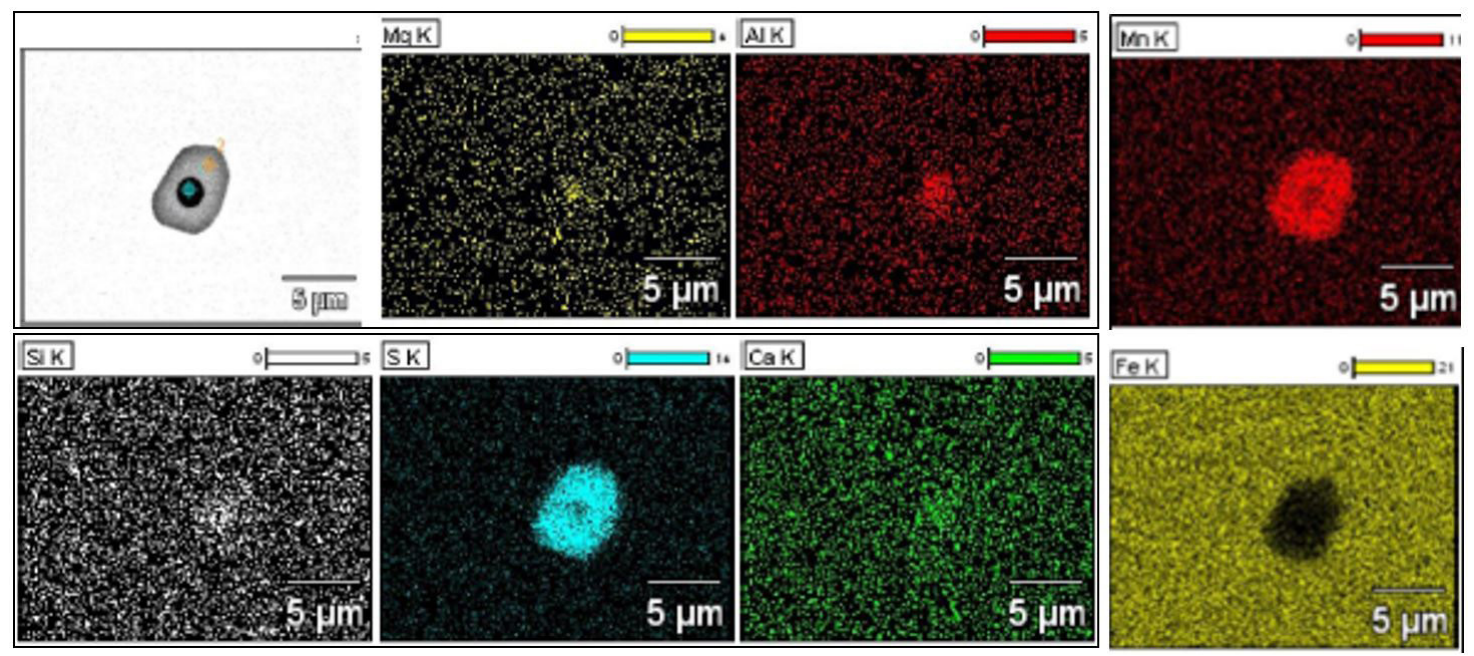

Figura 16. Mapeamento de raio-x realizado sobre a inclusão de sulfeto da Figura I5. As regiões em vermelho e azul correspondem ao Mn e o S respectivamente. 
Tabela 3. Composição química da inclusão da figura I 3 (\% massa)

\begin{tabular}{cccccccc}
\hline Elementos & $\mathbf{M g}$ & $\boldsymbol{A l}$ & $\mathbf{S i}$ & $\boldsymbol{S}$ & $\mathbf{C a}$ & $\mathbf{M n}$ & $\mathbf{F e}$ \\
\hline Ponto I & $\mathbf{1}, 69$ & 21,44 & 5,99 & 25,89 & 6,94 & 35,42 & 2,64 \\
\hline
\end{tabular}

Tabela 4. Composição química da inclusão da figura I4(\% massa)

\begin{tabular}{lccccccc}
\hline Elementos & $\mathbf{M g}$ & $\boldsymbol{A l}$ & $\mathrm{Si}$ & $\boldsymbol{S}$ & $\mathbf{C a}$ & $\mathbf{M n}$ & $\mathbf{F e}$ \\
\hline Ponto I & 3,08 & 24,92 & 5,43 & 24,48 & $6,5 \mathrm{I}$ & 30,10 & 5,48 \\
\hline
\end{tabular}

Tabela 5. Composição química da inclusão da figura I5 (\% massa)

\begin{tabular}{lccccccc}
\hline Elementos & $\boldsymbol{M g}$ & $\boldsymbol{A l}$ & $\boldsymbol{S i}$ & $\boldsymbol{S}$ & $\boldsymbol{C a}$ & $\mathbf{M n}$ & $\boldsymbol{F e}$ \\
\hline Ponto I & $\mathrm{I}, 5 \mathrm{I}$ & $\mathbf{I} 2,27$ & 4,83 & 28,34 & 8,52 & 40,30 & 4,23 \\
Ponto 2 & $0, \mathrm{II}$ & 0,47 & $0,3 \mathrm{I}$ & 36,62 & 0,99 & 54,20 & $7,3 \mathrm{I}$ \\
\hline
\end{tabular}

Portanto de acordo com os estudos de (Wakoh et al. [13]), verificou-se que os óxidos podem ser utilizados como sítios nucleantes de sulfetos. Não foram encontradas inclusões do tipo MA e CMA como núcleo para precipitados de MnS sugerido por Peng-ju Chen et al. [6], provavelmente pelo baixo teor de $\mathrm{Mg}$, pois se trata de uma aço de baixa liga. Além disso, o tratamento com Ca sugerido por Bielefeldt e Vilela [17] para aumento da janela líquida (devido à redução do ponto de fusão das inclusões complexas) deve ser controlado para não reduzir o teor de enxofre. Um controle aprimorado destes óxidos pode ser utilizado para uma melhor dispersão e controle no tamanho e morfologia dos sulfetos, devido ao fato que a maioria dos sulfetos nucleados eram do tipo III (angular) e irregular.

\section{CONCLUSÃO}

O presente trabalho propos o estudo das inclusões de $\mathrm{MnS}$ na sua morfologia, tamanho e distribuição. De acordo com os estudos abordados neste trabalho, a forma dos sulfetos podem variar de acordo com o teor de enxofre, o espaçamento interdendrítico e a taxa de resfriamento. Isto foi visto pela presença de sulfetos do tipo III (angular) e irregular na região de meio raio de um tarugo e sulfetos do tipo Il (eutético) e irregular eutético no núcleo das amostras analisadas. Algumas formas de inclusões não foram detectadas no aço analisado, como CMA. Neste caso, provavelmente pelo baixo teor de Mg no aço estudado. Entretanto, a morfologia das inclusões observadas no MEV estão de acordo com as apresentadas na revisão da literatura deste estudo.

A distribuição de sulfetos ao longo das micrografias processadas pelo aplicativo computacional demonstrou que o número de inclusões aumentou em direção ao núcleo, diminuindo o tamanho médio do diâmetro. Algumas inclusões tornaram-se mais alongadas no núcleo, comprovado pela redução da circulariedade destas. $O$ aumento do número de sulfetos e redução do tamanho da inclusão está associado ao aumento da região rica em enxofre devido ao aumento no espaçamento interdendrítico. Ainda existe um aumento da segregação no núcleo. Através disto há uma maior formação se sulfetos do tipo II (eutético) e irregular eutético, embora tenha sido constatado que o número de sulfetos do tipo III (angular) e irregular não tenha variado nas diferentes regiões.

Quanto à presença de óxidos sendo utilizados como sítios de nucleação de inclusões de sulfetos, os resultados obtidos neste trabalho condizem com os esperados, pois se verifica a correlação com a literatura. Como foi visto, muitos sulfetos estavam associados a inclusões do tipo III (angular). Pelas análises produzidas, foi observado através da análise química que óxidos compostos de alumínio e cálcio serviram como nucleantes de sulfetos de manganês. Fica a sugestão de trabalho futuro, o aprofundamento sobre o uso de óxidos para redução do tamanho dos sulfetos de manganês, principalmente alongados tipo 2 .

\section{Agradecimentos}

Agradeço aos Prof. Dr. Wagner Viana Bielefeldt e Prof. Dr. Antônio Cezar Faria Vilela pela dedicação e ensinamentos, além, dos colegas e ao Laboratório de Siderurgia (Lasid-UFRGS) pela oportunidade de defender meu mestrado.

\section{REFERÊNCIAS}

I Yamamoto K, Yamamura H, Suwa Y. Behavior of non-metallic inclusions in steel during hot deformation and the effects of deformed inclusions on local ductility. ISIJ International. 20 I I;5 I (I 2): I 987- I994. http://dx.doi.org/ I0.2355/ isijinternational.5 I. 1987.

2 Mapelli C, Nicodemi W, Vedani M, Zoppi A. Control of inclusion in a resulphurised steel. Steel Research. 2000;200(7I):I6I-I68. http://dx.doi.org/I0.1002/srin.200005707.

3 Oikawa Ol, Ohtani H, Ishida K, Nishizawa T. The control of the morphology of MnS inclusions in steel during solidification. ISIJ International. 1995;35(4):402-408. http://dx.doi.org// 0.2355/isijinternational.35.402.

4 Sims CE. The nonmetallic constituents of steel. Transactions of the Metallurgical Society of AIME. I959;2 I5:367393.

5 Li M, Wang F, Li C, Yang Z, Meng Q, Tao S. Effects of cooling rate and Al on MnS formation in medium-carbon non-quenched and tempered steels. International Journal of Minerals Metallurgy and Materials. 20I5;22(6):589-596. http://dx.doi.org/I0.1007/s I 26I3-0I5-IIII-I. 
6 Chen P, Zhu C, Li G, Dong Y, Zhang Z. Effect of sulphur concentration on precipitation behaviors of MnS-containing Inclusions in GCrI5 bearing steels after LF refining. ISIJ International. 2017;57(6): 1019-1028. http://dx.doi. org/I0.2355/isijinternational.ISIJINT-20I7-007.

7 Zhang $\mathrm{X}$, Zhang L, Yang W, Dong Y. Characterization of MnS particles in heavy rail steels using different methods. Steel Research International. 2017;88(I):I-16. https://doi.org/10.1002/srin.201600080.

8 Yu H, Kang Y, Zhao Z, Sun H. Morphology and precipitation kinetics of MnS in low-carbon steel during thin slab continuous casting process. Journal of Iron and Steel Research International. 2006; 13(5):30-36. http://dx.doi. org/I0.1016/S1006-706X(06)6009I-5.

9 Imagumbai M. Behaviors of manganese-sulfide in aluminum-killed steel solidified uni-directionally in steady state. Dendrite structure and inclusions. ISIJ International. 1994;34(I I):896-905. http://dx.doi.org/ I0.2355/ isijinternational.34.896.

10 Diederichs R, Bleck W. Modelling of manganese sulphide formation during solidification, Part I: description of MnS formation parameters. Steel Research International. 2006;77(3):202-209. http://dx.doi.org/I0. I002/srin.200606375.

I Chen Y, Yan W, Zhao A. Precipitation of AIN and MnS in low carbon aluminium-killed steel. Journal of Iron and Steel Research International. 2012;19(4):5I-56. http://dx.doi.org/I0.10I6/SI006-706X(I2)60087-9.

12 Zhang XF, Lu WJ, Qin RS. Removal of MnS inclusions in molten steel using electropulsing. Scripta Materialia. 2013;69(6):453-456. http://dx.doi.org/10.1016/j.scriptamat.2013.05.033.

13 Wakoh S, Sawai T, Mizoguchi S. Effect of S content on the MnS precipitation in steel with oxide nuclei. ISIJ International. 1996;36(8): I0I4-I02I. http://dx.doi.org/I0.2355/isijinternational.36. I0I4.

14 Ito M, Masumitsu N, Matsubara K. Formation of manganese sulfide in steel. ISIJ International. 198I;2I (7):477-484. http://dx.doi.org// 0.2355/isijinternational I 966.21.477.

I5 Luo S, Wang B, Wang Z, Jiang D, Wang W, Zhu M. Morphology of solidification structure and mns inclusion in high carbon steel continuously cast bloom. ISIJ International. 20I7;57(I I):2000-2009. http://dx.doi.org/I0.2355/ isijinternational.ISIJINT-2017-294.

16 Shao X, Wang X, Jiang M, Wang W, Huang F. Effect of heat treatment conditions on shape control of large-sized elongated MnS inclusions in resulfurized free-cutting steels. ISIJ International. 20I I;5 I(I2):I995-200I. http://dx.doi. org/I0.2355/isijinternational.5I. 1995.

17 Bielefeldt WV, Vilela ACF. Study of Inclusions in high sulfur, Al-Killed Ca-Treated steel via experiments and thermodynamic calculations. Steel Research. 2015;86:375-385.

I8 Imagumbai M, Takeda T. Influence of calcium-treatment on sulfide- and oxide-inclusions in continuous-cast slab of clean steel. Dendrite structure and inclusions. ISIJ International. 1994;34(7):574-583. http://dx.doi.org/10.2355/ isijinternational.34.574.

19 Ren Y, Zhang L, Li S, Yang W, Wang Y. Formation mechanism of CaO-CaS inclusions in pipeline steels. AlSTech Proceedings. 20I4:1607-1617.

$20 \mathrm{Y} . \mathrm{Hu}$, Chen WQ, Han HB, Bai RJ. Influence of calcium treatment on cleanness and fatigue life of 60Si2MnA spring steel. Ironmaking \& Steelmaking: Processes, Products and Applications. 20I7;44(I):28-36. http://dx.doi.org/I0. 1080 /03019233.2016.II 53026 .

Recebido em: 30 Nov. 2018

Aceito em: 16 Ago. 2019 